\title{
Review
}

\section{Separation methods for taurine analysis in biological samples}

\author{
Shifen Mou ${ }^{\mathrm{a}, *}$, Xiaojing Ding ${ }^{\mathrm{b}}$, Yongjian Liu ${ }^{\mathrm{a}}$ \\ ${ }^{a}$ Research Center for Eco-Environmental Sciences, Chinese Academy of Sciences, P.O. Box 2871, Beijing 100085, China \\ ${ }^{\mathrm{b}}$ Beijing Center for Disease Prevention and Control, Beijing 100013, China
}

\begin{abstract}
Taurine plays an important role in a variety of physiological functions, pharmacological actions and pathological conditions. Many methods for taurine analysis, therefore, have been reported to monitor its levels in biological samples. This review discusses the following techniques: sample preparation; separation and determination methods including highperformance liquid chromatography, gas chromatography, ion chromatography, capillary electrophoresis and hyphenation procedures. It covers articles published between 1990 and 2001.
\end{abstract}

(C) 2002 Elsevier Science B.V. All rights reserved.

Keywords: Reviews; Taurine

\section{Contents}

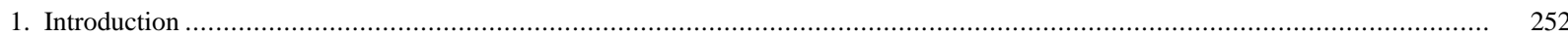

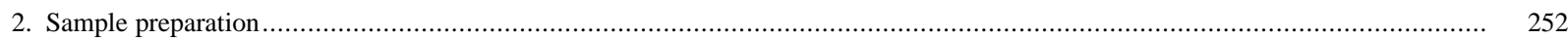

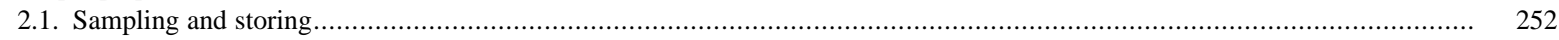

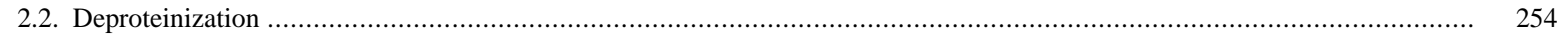

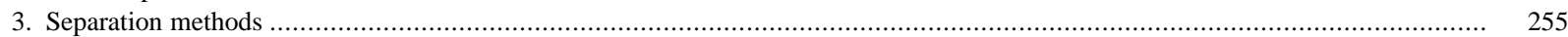

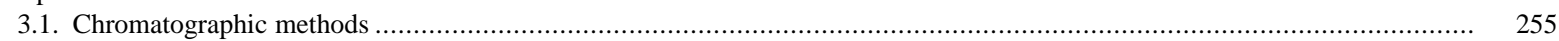

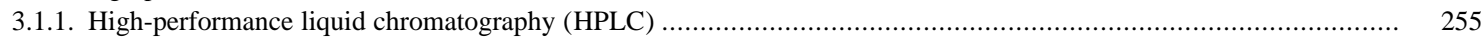

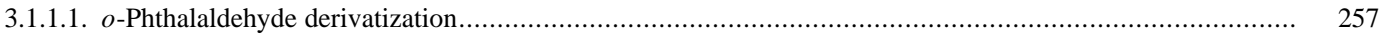

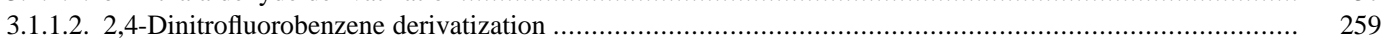

3.1.1.3. 1-Dimethylaminonaphthalene-5-sulfonyl chloride derivatization ........................................... 260

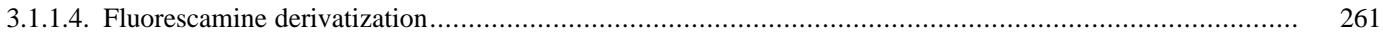

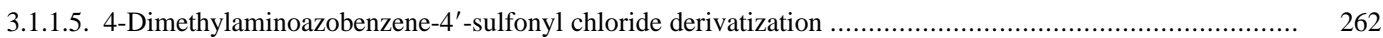

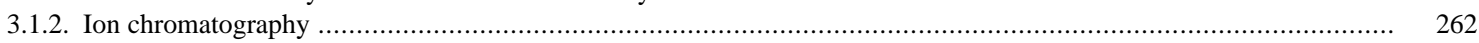

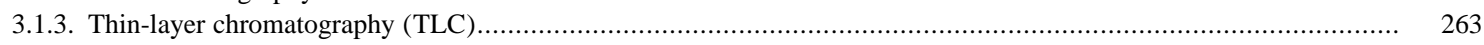

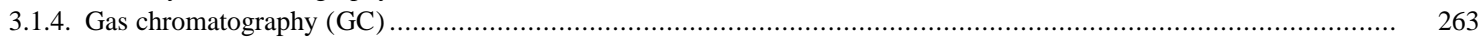

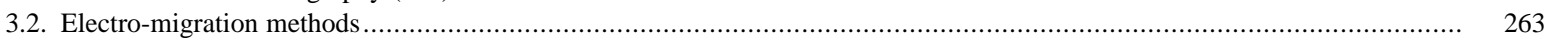

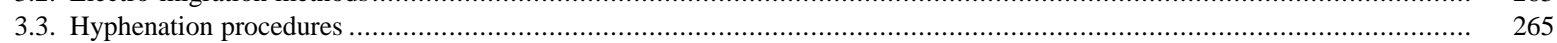

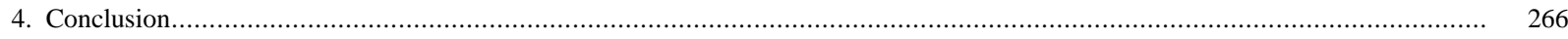

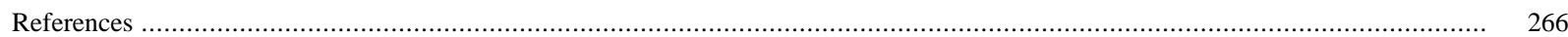

*Corresponding author. Tel./fax: +86-106-284-9239.

E-mail address: shifenm@mail.rcees.ac.cn (S. Mou).

1570-0232/02/\$ - see front matter (C) 2002 Elsevier Science B.V. All rights reserved. 


\section{Introduction}

Taurine (2-aminoethanesulfonic acid) is a free, conditionally essential, non-protein, sulfur-containing $\beta$-amino amino acid occurring widely in mammals' especially halobios' fluids and tissues. It is one of the final end-products of cysteine metabolism in mammals and is excreted in urine. Its levels varied significantly in different biological samples, ranging from high $\mathrm{m} M$ concentrations $(50-100 \mu \mathrm{g} / \mathrm{ml}$ in plasma) in several vital organs to a very low concentration in several tissues and physiological fluids $(1-50 \mu M)$. Considerable evidence has demonstrated that taurine not only has physiological functions, which is able to act as a neurotransmitter, antioxidant, modulator of intracellular calcium levels and osmolyte, etc., but also has pharmacological actions, which are able to protect the liver and benefit the gallbladder, to lower blood pressure and increase anti-arrhythmia, etc. Its pathological functions show that changes of taurine levels in physiological fluids and tissues also has a close relationship with many diseases such as Alzheimer's disease, cardiovascular diseases, hypercholesterolemia, epilepsy and other seizure disorders, etc. So, clinically, taurine has been used with varying degrees of success in the treatment of a wide variety of conditions, including: cardiovascular diseases, hypercholesterolemia, epilepsy and other seizure disorders, macular degeneration, Alzheimer's disease, hepatic disorders, alcoholism, and cystic fibrosis. However, the use of taurine as a drug to treat specific diseases has been limited. Thus, analytical methods with high sensitivity, selectivity and rapidity for precise assay of taurine are required, and the results can be used to diagnose diseases.

Taurine is found in high concentrations at birth, decreasing rapidly with age [1]. Human, nonhuman primate species and cats have a very limited ability to synthesize taurine, so they are prone to develop taurine deficiency and largely dependent on an exogenous source. Patients with depleted taurine concentrations may suffer from immunodeficiency, cardiomyopathy, retinal degeneration and growth retardation, especially if deficiency occurs during development [2,3]. All these findings have encouraged the use of taurine in infant formula, nutritional supplements and energy promoting drinks. Taurine as a legal food additive has now been widely used in many countries. Nonetheless, some researches show that taurine of the right amount can protect liver from free radicals, and excess taurine will do harm to liver [4]. A reliable method to analyze taurine in foods for quality control purpose is required. The results can be used to evaluate nutritional significance of taurine. Since taurine analysis is one of the fundamental measurements of biological sciences, with applications in every aspect of biological research, clinical medicine, biotechnology (agriculture, medicine) and food technology, depending on the purpose of analysis, and driven increasingly by a need for higher sensitivity, analysis of taurine in various biological samples is an active area at present.

Taurine is an organic weak acid with dissociation constant $\mathrm{p} K_{\mathrm{a}}=4.96$. It is an amphoteric compound and is easily soluble in water, and can be extracted directly with water. It keeps stable in the process of cooking because of its high melting point $\left(>300^{\circ} \mathrm{C}\right)$. It also remains stable in acids and bases. It has no ultraviolet chromophore and relies on derivatization to permit ultraviolet or fluorescence detection.

Based on these physicochemical properties, many separation methods for taurine analysis in biological samples have been reported. In this paper, only the developments since 1990 in separation methods for taurine analysis are reviewed.

\section{Sample preparation}

The most challenging thing in taurine analysis is that taurine is present at low concentrations in complex biological samples. The assay of taurine therefore may suffer from interferences of biological matrices. In addition, the concentrations of taurine in some samples were variable. So, developing a suitable procedure for the collection and pretreatment of various biological samples was the first step for a veracious determination of taurine.

\subsection{Sampling and storing}

The concentration of taurine in biological samples was not only affected by the health condition, dietary habit [5], but also by the collecting time, sampling 
mode, pretreatment and storage method, etc. For example, blood samples should be pretreated immediately after collection. For this reason, how to sample and store the samples without being contaminated was the first factor for the accurate determination of taurine in all kinds of biological samples.

For powdery and granular samples, such as milk powder [6], infant formula [7], medicine [8], etc., it is as simple as dissolving the samples in pure water at a suitable concentration. Then, the sample could be used for the next treating process or stored at $4{ }^{\circ} \mathrm{C}$ until analysis.

Most liquid samples are physiological ones, such as urine, cerebrospinal fluid (CSF) and blood (including plasma and serum, etc.). For urine, all $24 \mathrm{~h}$ samples should be collected. For some animals, emiction does not occur every day, therefore a midtime of 2 days should be used as reference time. If there is some hair or food debris in the sample, it must be removed in the first place by centrifugation [5]. When urine was analyzed by ion chromatography, the sample pretreatment was as simple as dilution [8]. As for high-performance liquid chromatography (HPLC) techniques, the urine sample must be deproteinized directly either by adding some protein precipitant [9] or be diluted with buffer, and then adjusted to nearly neutral prior to the deproteinization process [10]. With mass spectrometry (MS) detection, urine sample could be hydrolyzed with $12 \mathrm{~mol} / 1$ hydrochloric acid and then purified by anion/cation-exchange chromatography [11]. If a urine sample could not be treated in time, it should be stored frozen at $-24{ }^{\circ} \mathrm{C}$ until assay [9].

When a CSF sample was collected, much care should be taken to make the sample free of blood. Since CSF is quite "clean", derivatization could be done directly on the sample without any pretreatment if they were diluted enough prior to derivatization $[12,13]$. However, the dilution step itself may introduce contaminants, which interfere with the assay of taurine at sub-picomol levels and may produce incomplete derivatization. Moreover, after a few runs, strongly adsorbed compound accumulated on the top of the column, affecting precision and accuracy of chromatographic performance [13]. Therefore, the CSF sample should be deproteinized immediately and the supernatant be used for derivatization or stored at $-20 /-80{ }^{\circ} \mathrm{C}$. Anesi et al.
[14] compared the differences between the untreated CSF sample and the just deproteinized sample. They found that when samples were stored at -20 or $-80{ }^{\circ} \mathrm{C}$ for $>14$ days, the concentration of taurine showed a significant decrease in comparison with the content of taurine in the fresh aliquots. However, when samples were deproteinized and centrifuged, the clear supernatants were immediately adjusted to $\mathrm{pH} 7.3$ with sodium bicarbonate and stored at -20 or $-80^{\circ} \mathrm{C}$, no significant difference was found in taurine concentrations of the CSF sample during the 30-day period. Therefore, In order to avoid artificial changes of taurine concentrations caused by in vitro modifications of amino acids and the tendency for an increased taurine value at high temperature in CSF sample, the authors suggested that native CSF should be stored for no longer than a few hours. If CSF samples could not be determined in a very short period, it was strongly recommended to deproteinize samples immediately following the sampling and neutralize the supernatant of the acidified CSF after centrifugation. Then the sample could be stored at -20 or $-80^{\circ} \mathrm{C}$ [13] for a relatively long time.

As for blood samples, the technique of blood collection will not give serious problems for the quantitation of taurine. However, the time of blood withdrawal and the allied problem of dietary intake may be another reason for varying results between laboratories. Fortunately, this could be standardized by taking blood samples between 08.00 and $09.00 \mathrm{~h}$ after overnight fasting. Most laboratories collected blood by venipuncture into tubes containing anticoagulant heparin [15], potassium ethylenediaminetetraacetic acid [16] or sodium citrate [17] and then centrifuged them into several parts such as plasma and serum with various centrifugal conditions. When the sample was collected with narrow-bore syringe needle and centrifuged at high speeds, platelet (which contain high concentrations of taurine) would be ruptured and platelet-poor plasma was obtained. So, care should be taken to prevent hemolysis, which would lead to false increase in the concentration of taurine. However, when the sample was taken using a large-bore butterfly syringe and centrifuged at no more than $150 \mathrm{~g}$, it was possible to avoid rupture of the platelet and platelet-rich plasma could be acquired [18]. Therefore, a large-bore butterfly syringe was a better choice to avoid cell damage [17]. 
Following the centrifugation, the sample should be deproteinated immediately or stored at $-80{ }^{\circ} \mathrm{C}$ until analysis [16].

With regard to tissue samples such as liver [19], brain [20], kidney [21] and heart [10], they must be immediately homogenized and extracted in a homogenizer with glass pestle after the collection, and then deproteinize the extracting solution. The solutions used for homogenization and extraction include borate buffer [22], phosphate buffer [10], sucrose solution [21] mixed solution (water-sodium tungstate-sulfuric acid, 7:1:1, v/v) [23], acetate buffer [24], methanol [25] and ethanol [26], etc. Aristoy and Toldrá [27] compared the recoveries of taurine extracted from fresh pork muscle and drycured ham with $0.03 \mathrm{~mol} / 1$ phosphate buffer $(\mathrm{pH}$ 7.4), $0.05 \mathrm{~mol} / 1$ acetate buffer $(\mathrm{pH} 4.5)$ and 0.1 mol/1 hydrochloric acid. The results indicated that the highest taurine recoveries was obtained by using $0.1 \mathrm{~mol} / 1$ hydrochloric acid. Boiling water could also be used to extract taurine from tuna or squid meat [28]. In order to get the maximal recovery during the homogenization process, the time of homogenization should be well controlled. Homogenization for $8 \mathrm{~min}$ was enough to get the maximum yield [27].

\subsection{Deproteinization}

Removal of protein from the sample is a prerequisite for the accurate detection of taurine at sub-picomol levels, derivative formation and the precision as well as accuracy of chromatographic performance. Both ultrafiltration and protein precipitation techniques have been reported for the accurate assay of taurine in various biological samples. The widely used protein precipitation reagents include mixed solution, organic solvents (acetonitrile, methanol), acids [sulfosalicylic acid (SSA), perchloric acid, trichloroacetic acid (TCA)], etc. The advantages and disadvantages of these techniques are discussed below.

Ultrafiltration is a physical deproteinization technique. Aristoy and Toldrá [27] tested the efficiency of $1.65 \cdot 10^{-20}$ and $1.65 \cdot 10^{-21} \mathrm{~g}$ cutoff membranes for the filtration of pork muscle extracting solution. The recoveries of taurine with the $1.65 \cdot 10^{-20}$ and $1.65 \cdot 10^{-21} \mathrm{~g}$ membranes were 99.1 and $101.6 \%$, respectively. Nicolas et al. [7] reported that the sample concentrations and viscosities would not affect recoveries of taurine in the ultrafiltration process, taurine was not retained on the filter in this experiment and the spinning time is not a critical factor affecting the results. It only needed to prolong the spinning time so as to get sufficient sample filtrate, which is inconvenient for routine analysis. Kelly et al. [18] made a comparison of acetonitrile precipitation and ultrafiltration for the deproteinization of plasma. They found that in order to recover a usable volume of filtrate, the ultrafiltration cartridges $\left(1.98 \cdot 10^{-20}\right.$ cut-off filter) were spun at $2000 \mathrm{~g}$ for 90 min. However, with the acetonitrile precipitation method, the recovery of taurine was $99.2 \%$, and the method was more expediently to execute than the costly and lengthy ultrafiltration method. McMahon et al. [17] reported that few of the lower molecular mass interferents were removed during the ultrafiltration. So, the ultrafiltration method was not widely used.

A mixed solution of water, sulfuric acid and sodium tungstate was used for deproteinization of urine, plasma and homogenate of liver [24,29]. Excellent recoveries could be obtained. However, it was a little complex to prepare the solution, this method therefore did not appear to have been widely adopted.

Tcherkas et al. [30] found that protein precipitation with methanol was most suitable in combination with a mobile phase containing $18 \%$ methanol. Marks et al. [16] reported that after deproteinization with methanol and derivatization with pentafluorobenzoyl chloride, perfect recovery of taurine in plasma could be obtained.

Acetonitrile was a widely used solvent for the deproteinization of biological samples. Uhe et al. [31] compared the efficacy of acetonitrile, TCA and SSA as deproteinizing agents. The results indicated that the three reagents had almost the same capacity for deproteinization when taurine was determined. Aristoy and Toldrá [27] investigated the efficiencies of acetonitrile, perchloric acid, SSA and TCA for the deproteinization of muscle extracts. The results indicated that acetonitrile was the best one in these protein precipitants. McMahon et al. [17] suggested that deproteinization by acetonitrile proved to be the most facile and reproducible method of sample preparation and was the easiest to execute. 
Rizzo et al. [13] evaluated the effects of several precipitating agents on the levels of taurine in CSF. It was found that acid agents resulted in a twofold overestimate of free taurine. However, taurine was stable in organic solvents. With low amount of TCA, more than $99 \%$ protein could be removed and protein-free supernatant could be easily got. So, they considered TCA as the optimum protein remover for biological samples, which was also confirmed by other workers [14]. In addition, perfect recovery $(97.8 \%)$ of taurine was obtained [2].

Perchloric acid proved to be another efficient deproteinization reagent for many biological samples, such as the homogenate of human heart muscle biopsies [10], plasma [32], tissues [20,22,33], etc. However, McMahon et al. [17] considered that when perchloric acid was used as protein remover, it was subsequently difficult to raise the $\mathrm{pH}$ reproducibly prior to derivatization.

As a protein-precipitating agent, SSA has got many applications in various biological samples, such as urine [9], plasma [15], human breast milk [3], serum [12], tissue homogenate [19], etc. Waterfield [5] reported that the use of SSA was an effective method, which could result in a higher recovery of taurine from tissue homogenates than perchloric acid or TCA. Ferreira et al. [3] carried out recovery studies on two infant formulae samples, which had very high and very low concentrations of taurine, respectively. The results indicated that the mean recoveries of spiked taurine at three different concentrations in the two samples were 98.8 and $99.2 \%$, respectively. So, it could be concluded that irrespective of some problems encountered by several investigators, SSA still was the most widely used precipitant for the deproteinization of biological samples.

When a biological sample contains large amounts of amino acids other than taurine, which may interfere with the determination of taurine, purification procedures by a cation-exchange column (Dowex 50W, $2.0 \mathrm{ml}, 5.5 \times 0.7 \mathrm{~cm} \mathrm{I.D.,} \mathrm{H}^{+}$form) [35] or an ion-exchange column filled with both an anionic and cationic resin (Amberlite AG1 $\times 8,200-$ 400 mesh, $0.5 \mathrm{ml}$ and Amberlite AG50W- $\times 8,200-$ 400 mesh, $1 \mathrm{ml}$ ) have been proved practical. Taurine could be washed out with water and good recoveries could also be obtained [5,11]. Unfortunately, extra impurities could be introduced in the samples. On this account, the method was only used in a limited range.

The sample pretreatment methods for typical samples are listed in Table 1.

\section{Separation methods}

\subsection{Chromatographic methods}

\subsubsection{High-performance liquid chromatography (HPLC)}

Before 1990, taurine determinations had been carried out mainly by means of various automated ion-exchange post-column derivatization (mostly ninhydrin) methods, namely the amino acid analyzer. The specificity and sensitivity for taurine in this system are not always satisfactory, and the chromatography is often affected by coeluting compounds. In addition, the process is time consuming (particularly if determination of all the other amino acids is also required) and expensive. The derivatization reagents must be kept under nitrogen protection, which is inconvenient for routine analysis.

With HPLC techniques, the number of articles using the amino acid analyzer decreased [7,36-39]. Developments involving reversed-phase (RP) HPLC have attempted to avoid some of the inconveniences of all above-mentioned approaches, predominantly utilizing $o$-phthalaldehyde (OPA) pre-column derivatization procedures. RP-HPLC either in isocratic or gradient elution modes has been routinely employed with much success and seems to be the method of choice for determination of taurine in the greatest variety of biological samples due to its high sensitivity and flexibility. The published HPLC methods outnumber all other separation methods. The bulk of these are relatively involving pre- or post-column derivatization followed by ultraviolet, fluorescence and/or electrochemical detection to improve both the quality of separation as well as detection sensitivity. An obvious advantage of precolumn derivatization relatively to post-column derivatization is that it requires a basic HPLC system without extra reagent pumps, mixing manifolds and reaction coils, etc. So, pre-column derivatization HPLC methods are more often applied when analyz- 
Table 1

The sample pretreatment and separation methods for the typical biological samples

\begin{tabular}{|c|c|c|c|c|c|c|c|c|}
\hline Sample & Collection and preparation & Deproteinization & Storage $\left({ }^{\circ} \mathrm{C}\right)$ & Derivatization & Instrument & Separation & Recovery (\%) & Ref. \\
\hline Oyster powder & Dissolved & - & - & DNFB & HPLC-UV detector & $\mu$ Bondapak Phenyl column & 100.8 & [57] \\
\hline Urine & 24-h samples collected, diluted, centrifuged & Ion-exchange resin & $-20 /-80$ & OPA & HPLC-fluorescence detector & LiChrospher 100 RP-18 column & $99.59 \pm 2.13$ & [5] \\
\hline Tissue & Homogenized with perchloric acid & Perchloric acid & -80 & OPA & HPLC-electrochemical detector & $\mathrm{C}_{18}$ IP Ultrasphere column & $107.4 \pm 1.3$ & [20] \\
\hline CSF & Centrifuged & TCA & -80 & OPA & HPLC-electrochemical and fluorescence detector & $\mathrm{C}_{18}$ Rosil HL & - & [13] \\
\hline Human milk & Centrifuged & SSA & -40 & DNFB & HPLC-UV detector & $\mu$ Bondapak Phenyl column & 95.8 & [34] \\
\hline Plasma & Centrifuged & Acetonitrile & -70 & OPA & HPLC-fluorescence detector & LiChrospher $100 \mathrm{C}_{18}$ column & 105 & [31] \\
\hline Serum & Centrifuged & SSA & -80 & OPA & HPLC-fluorescence detector & LiChrospher 100 RP-18 column & - & [5] \\
\hline
\end{tabular}


ing taurine in complex biological samples. Two postcolumn methodologies using thiamine have also been reported $[29,40]$. As different derivatization reagent will vary in reaction rate and stability, on-column fluorescence derivatization for the determination of taurine in foods was reported [41]. On-column reactions provided a means to obtain reproducible labeling conditions, which enabled one to obtain precise analytical results.

Usually, HPLC methods developed for amino acid analysis could be applied to the determination of taurine without any modification $[9,15,19,24,25$, $30,31,33,42-44]$. The main disadvantage of these techniques was poor selectivity. In some cases, taurine was more concerned than all the other amino acids. Therefore, rapid and sensitive HPLC methods for only taurine (or small group) analysis in biological samples are required to solve particular problems. Since derivatization techniques play a key role in getting higher sensitivity, shorter analysis time and better resolution of complex biological samples, some derivatization reagents with sensitivity, stability, selectivity, rapidity and simplicity are investigated in detail for analysis of taurine. Typical reagents include OPA, 2,4-dinitrofluorobenzene (DNFB), 1-dimethylaminonaphthalene-5-sulfonyl chloride (dansyl-Cl), 4-dimethylaminoazobenzene$4^{\prime}$-sulfonyl chloride (DABS-Cl), fluorescamine and thiamine, etc. The features of these pre-column derivatization methods for taurine analysis are shown in Table 2. To be convenient, the published HPLC separation methods were discussed according to the derivatization reagents they used.
3.1.1.1. o-Phthalaldehyde derivatization. OPA, introduced as early as 1971, is probably the most commonly used in RP-HPLC for the determination of free amino acids including taurine in complex biological samples, such as tissue homogenates, urine, infant formulae and serum, etc. [5,13,20,4548]. OPA reacts with taurine in the presence of a reducing agent, such as 2-mercaptoethanol (2-ME) [5,13,46-49], urea [51] or sodium sulfite [52] to form a substituted isoindole ring. The derivative not only has a strong absorbance at 260 and $340 \mathrm{~nm}$ but also a strong fluorescence at $475 \mathrm{~nm}$, which was at least 20 times more sensitive than ninhydrin [45]. Most importantly, the unreacted OPA reagent does not have the fluorescent characteristics, a property known as fluorogenic. In addition, the derivative is also electroactive which could be detected with electrochemical detection. These properties of OPA have proved useful for the analysis of taurine in various biological samples by HPLC methods.

Short RP-HPLC columns packed with 3- or 5- $\mu \mathrm{m}$ particles have been developed for the analysis of taurine. Murai et al. [20] used a combination of a very short column $(45 \times 4.6 \mathrm{~mm}$ I.D. $)$ and higher flow-rate $(2.7 \mathrm{ml} / \mathrm{min}), 30{ }^{\circ} \mathrm{C}$ column temperature, methanol $(10 \%)$, and tetrahydrofuran $(12 \%)$ concentration, which was different from those generally used. The results showed that a short column packed with smaller particle size (3 or $5 \mu \mathrm{m}$ ) had a remarkable advantage that permitted shortening of an analysis time without an obvious loss of separation efficiency of the column. The determination of taurine in rat brain tissues by electrochemical de-

Table 2

Summary of pre-column derivatization methods used for taurine analysis by HPLC

\begin{tabular}{llllll}
\hline & OPA & DNFB & Dansyl-Cl & DABS-Cl & Fluorescamine \\
\hline Derivatization time (min) & $<1$ & 30 & 30 & $10\left(70{ }^{\circ} \mathrm{C}\right)$ & Instantaneous \\
Sample preparation & Very simple & Complex & Simple & Simple & Simple \\
Automation of derivatization & Yes & No & No & No & Yes \\
Removal of reagent by drying & No & Yes & No & No & No \\
Quantitative yield & Yes & Yes & Yes & Yes & Yes \\
Stable derivative & No & Yes & Yes & Yes & No \\
Interfering side-products & No & Yes & Yes & No & Fluor \\
Detection & Fluor & $360 \mathrm{~nm}$ & Fluor & Plom & Picomol \\
Sensitivity & Femtomol & Picomol & Picomol & Picomol Good & Good \\
Reproducibilty & Very good & Good & Good & Good & \\
\hline
\end{tabular}

Abbreviations: $\mathrm{OPA}=o$-phthaldialdehyde; $\mathrm{DNFB}=2$,4-dinitrofluorobenzene; dansyl-Cl=1-dimethylaminonaphthalene-5-sulfonyl-chloride; DABS- $\mathrm{Cl}=4$-dimethylaminoazobenzene-4' ${ }^{-}$-sulfonyl chloride; fluor=fluorescence. 
tection could be completed within an analysis time of only $2 \mathrm{~min}$ for each chromatographic run. Thus, permitted the determination of up to 100 samples per working day. Moreover, the detection limit of the method was 0.1 pmol for taurine and was comparable to the lowest value $(0.04-0.08 \mathrm{pmol})$ previously detected by HPLC methods. However, with complex amino acid mixtures containing components such as leucine, valine and tryptophan, the separation based on this technique took much longer.

Waterfield [5] proposed that levels of urinary taurine might provide a useful non-invasive maker of hepatotoxicity and perturbations in sulfur amino acid and protein metabolism as these conditions alter urinary and liver concentrations of taurine. He utilized dual-bed Dowex resins to extract taurine and remove all interfering amino acids. The cleaned samples were then derivatized by mixing equal volumes of OPA-2-mercaptoethanol for $1.5 \mathrm{~min}$ prior to injection onto a $\mathrm{C}_{18}$ column. Since the fluorescence of the adduct decayed with time, the timing of the injection must be kept constant. Isocratic elution of the adducts was carried out using $\mathrm{NaH}_{2} \mathrm{PO}_{4}(0.05 M, \mathrm{pH}$ 5.4) in methanol-water (43:57, v/v). Homoserine was used as an internal standard to facilitate the standardization and quantitation of samples and the run time was within $6 \mathrm{~min}$ with homoserine and taurine eluting after 3 and 4 min, respectively. Low concentration to 0.5 pmol of taurine on the column could be detected. When the taurine levels were higher ( $1 \mathrm{nmol}$ or more on the column, e.g., in rat urine samples), it was possible to use UV absorbance detection at $350 \mathrm{~nm}$. Ferreira et al. [3] proposed a similar HPLC procedure for routine and selective determination of taurine in infant formulas and breast milk. The samples were deproteinized and filtered through W42 paper. Afterwards, taurine was derivatized with OPA-2-mercaptoethanol. The derivatization reaction was allowed to proceed for exactly $1.5 \mathrm{~min}$, at that time an aliquot was injected onto a reversed-phase column $\mathrm{C}_{18}$ $\left(\mathrm{S}_{10} \mathrm{ODS}_{2}\right)$. Isocratic elution was carried out using $0.05 \mathrm{~mol} / \mathrm{l}$ phosphate buffer, $\mathrm{pH}$ 5.3-methanol (60:40) mixture. The effluent was then detected at $350 \mathrm{~nm}$. The chromatographic run time of $5 \mathrm{~min}$ was comparable with the lowest reported time for fluorimetric determination and less than the run time reported for UV determination. No interference of other amino acids was observed.
Yang and Zhang [46] separated taurine from food by HPLC on a $\mathrm{C}_{18}$ ( $\mu$-Bondapak) column using methanol-acetone-water (the ratio was not given) as mobile phase and detected at $330 \mathrm{~nm}$. They also investigated the stability of the taurine derivatives. They claimed that the higher concentration of borate buffer, the more stable of the derivatives; the lower concentration of taurine, the faster decomposition of taurine derivatives. Zheng and Lin [47] determined taurine in eleven kinds of foods by HPLC on a $\mathrm{C}_{18}$ (Zorbax ODS) column using a mobile phase of 0.1 mol/1 $\mathrm{NaH}_{2} \mathrm{PO}_{4}$-methanol (40:60) buffer ( $\mathrm{pH} 4.9$ ). L-Glutamine was used as an internal standard. The taurine level in oyster was reported to be $10.2 \mathrm{mg} / \mathrm{g}$, which was the highest among the above eleven kinds of foods. The samples were cleaned by dual-bed ion-exchange resins, and then derivatized by mixing equal volumes of OPA-2-mercaptoethanol for $3 \mathrm{~min}$ to form an amino acid adduct which was monitored at $340 \mathrm{~nm}$ by UV detection. The detection limit was $12 \mathrm{ng}$ of taurine.

The analytical results using pre-column derivatization HPLC methods for the analysis of free amino acids in CSF were found to be discordant among various researchers. Rizzo et al. [13] suggested that the choice of the analytical methodology was the primarily responsible for the discrepancies among the published data. Severe criteria for choice of several parameters, e.g., ionic strength of mobile phase, $\mathrm{pH}$, concentration of organic solvent and column temperature must be considered for a suitable chromatographic separation. They gave a systematic evaluation of basic parameters (e.g., age, sex, severity of disease and CSF sample storage) to obtain valid reference values and developed a highly sensitive chromatographic method for $\gamma$-aminobutyric acid (GABA), aspartate (Asp), glutamate (Glu), glycine (Gly) and taurine (Tau) determination in the CSF. The separation of OPA derivatives could be completed by $25 \mathrm{mM}$ potassium phosphate $(\mathrm{pH}$ $5.9 \pm 0.02)-15 \%$ acetonitrile in isocratic elution within $55 \mathrm{~min}$. Other identified compounds, such as, glutamine, asparagine, histidine, citrulline, threonine and arginine were found not interfering. In addition, electrochemical detection coupled with fluorimetric detection of OPA derivatives offered improved versatility and specificity. It was recommended to analyze freshly collected CSF as described in Section 2.1. The CSF samples for the calculation of the 
reference values were taken from 40 healthy subjects, hospitalized for lumbar disk herniation, placed on the same diet and kept drug-free for at least 1 week. The mean values $(\mu \mathrm{mol} / 1)$ were: $0.27,0.62$, 5.32, 6.16, 0.16 for Asp, Glu, Gly, Tau and GABA, respectively.

In general, the derivatization reaction between taurine and OPA requires the presence of a thiol such as mercaptoethanol to ensure that derivatization occurs instantaneously. Unfortunately, the fluorescent isoindoles are rather unstable, which influences the accuracy and reproducibility of taurine analysis. The stability of the OPA-2-ME taurine adducts is shown in Fig. 1 [30]. However, with the advent of microdialysis sampling technique, automated on-line derivatization techniques make the instability being of less importance. Bianchi et al. [48] used OPA derivatization and fluorescence detection for the simultaneous determination of four amino acids (aspartate, glutamate, taurine and 4-aminobutyric acid) in perfusates obtained either in vitro, from superfusion of tissue slices, or in vivo from microdialysis experiments. The chromatographic run-time was short $(15 \mathrm{~min})$ enough to allow the analysis of microdialysis samples "on line", as they were collected during the experiment. This method was applied to the measurement of the basal and $\mathrm{K}^{+}$- or veratridine-stimulated release of these four amino acids from perfused neostriatal and nigral tissue slices and in the extracellular fluid during microdialysis of several brain areas. The apparatus were not given. Yang et al. [49] combined a microdialysis perfusion technique with a HPLC system equipped with a fluorescence detector to automatically monitor

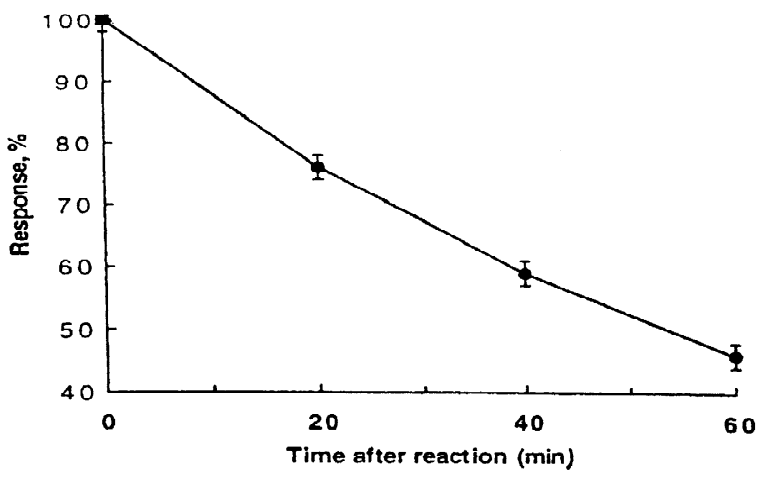

Fig. 1. The stability of the OPA-2-ME taurine adduct (response after 3 min was taken as $100 \%$ ). (Taken from Ref. [30] with permission). six brain extracellular amino acids (aspartate, glutamate, glutamine, glycine, taurine and $\gamma$-aminobutyric acid) in anesthetized rat. The microdialysate was on-line derivatized with OPA through a mixing tee prior to the injection onto the HPLC column. The efficiency of this on-line derivatization was equivalent to that performed with an off-line manner. Piepponen and Skujins [50] developed a rapid stepgradient HPLC method for determination of Glu, Gly and Tau in striatal microdialysates. The amino acids were first pre-column derivatized with OPA-2-mercaptoethanol by using an automated refrigerated auto-injector, and then separated on a new kind of non-porous ODS-C HPLC column with $3 \mu \mathrm{m}$ particle size followed by a wash-out step of late-eluting amino acids. Good resolution and sensitivity for Glu, Gly and Tau was thus obtained in less than $5 \mathrm{~min}$. Even with the wash-out step the analysis time remained less than $12 \mathrm{~min}$, which solved the problems with the stability of OPA-2-MCE amino acid derivatives.

Because of the toxicity [51] and pungent odor [52] of mercaptoethanol, alternatives to the use of mercaptoethanol have been reported. Xie and Zhang [51] used urea-OPA at $\mathrm{pH} 6.8$ instead of the commonly used mercaptoethanol-OPA under basic condition $(\mathrm{pH}$ 9.5) for the analysis of taurine in Lycium barbarum L. The derivative has a maximum absorption at $330 \mathrm{~nm}$, which avoid the interference of the derivatization reagent. The determined taurine level $(3.21 \mathrm{mg} / \mathrm{g})$ was in agreement well with an amino acid analyzer $(3.35 \mathrm{mg} / \mathrm{g})$. Rowley et al. [52] used sulfite-OPA under acidic conditions ( $\mathrm{pH} 4.5)$ instead of the commonly used mercaptoethanol-OPA under basic condition ( $\mathrm{pH}$ 9.5) for the analysis of amino acid neurotransmitters including taurine in CSF. The $\mathrm{N}$-alkyl-1-isoindole sulfonate derivatives were electroactive and odorless. It was stable at room temperature for up to $30 \mathrm{~min}$ and up to $5 \mathrm{~h}$ if kept on ice, which could be separated on a Rainin Dynamax $\mathrm{C}_{18}$ column in isocratic elution and then measured by electrochemical detection. The method was sensitive enough for the simultaneous determination of glutamate, glycine, taurine and $\gamma$-aminobutyric acid.

3.1.1.2. 2,4-Dinitrofluorobenzene derivatization. Taurine reacts with DNFB to form DNP derivative with UV absorbance at 254 and $350 \mathrm{~nm}$, respectively. 
The derivative once formed is stable in the reaction mixture at room temperature for at least 3 days. The possibility of extracting DNP derivatives from other interfered compounds and simplifying the chromatographic separation conditions makes this derivatization technique very attractive. However, the derivatization reaction usually takes more than $1 \mathrm{~h}$ at $40{ }^{\circ} \mathrm{C}$ and thus is not suitable for the rapid assay of large numbers of samples.

The derivatization catalyzed by dimethyl sulfoxide (DMSO, $0.5 \mathrm{ml})$ in a weakly-alkaline buffer $(0.01$ mol/1 $\mathrm{NaHCO}_{3}$, pH 9.0) was reported to be complete in $10 \mathrm{~min}$ at $40{ }^{\circ} \mathrm{C}$ to form their derivatives (DNP-taurine), which was suitable for the rapid assay of large number of samples. The derivative was stable for several days and excess DNFB did not interfere with the determination of taurine. Using this derivatization reaction, Chen et al. [34] accurately determined taurine concentrations in milk and urine over a wide linear range $(10-80 \mu \mathrm{g} / \mathrm{ml})$ by LC on a $\mu$-Bondapak Phenyl column at $40{ }^{\circ} \mathrm{C}$ with acetonitrile-water $(1: 1)$ and $0.01 \mathrm{~mol} / 1$ phosphate buffer (pH 5.0) as mobile phase in gradient elution (Fig. 2).

Xie et al. [53] reported that the derivatization reaction, without catalysis of DMSO, was complete in $60 \mathrm{~min}$ at $60^{\circ} \mathrm{C}$ in the dark. Then, the derivative was analysed on a Spherisorb $\mathrm{C}_{18}$ column with phosphate buffer solution of $\mathrm{pH} 7$-aqueous $50 \%$ acetonitrile $(7: 3)$ as mobile phase and detected at $360 \mathrm{~nm}$. The taurine levels in powdered oyster and clam were 70 and $66.7 \mathrm{mg} / 100 \mathrm{~g}$, respectively. Tang et al. [54] used the same derivatization conditions as

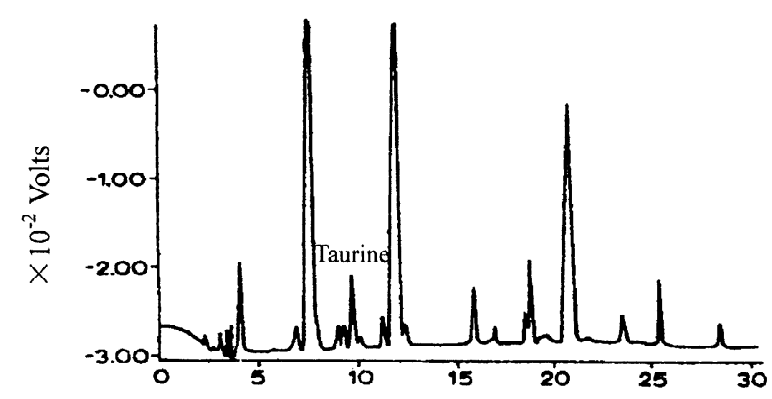

Fig. 2. HPLC chromatogram of taurine in human milk. Column: $\mu$ Bondapak phenyl column (30 $\mathrm{cm} \times 3.9 \mathrm{~mm}$ I.D.); column temperature: $40^{\circ} \mathrm{C}$; mobile phase: acetonitrile and phosphate buffer in gradient mode; detection: UV at $360 \mathrm{~nm}$. (Taken from Ref. [34] with permission). used by Xie et al., but a different LC column to determine industrial synthetic taurine and its intermediate product (2-aminoethyl sulfate) by HPLC. The derivatives were separated on a Supelcosil LC18 column with acetonitrile-water-phosphate buffer of $\mathrm{pH} 7$ (1:3:14) as mobile phase and detected at 360 $\mathrm{nm}$.

Polanuer et al. [28,55] established a rapid HPLC method for the determination of taurine in biological samples utilizing a different derivatization procedure from the above procedures $[34,51,53,54]$. The biological sample, after extracted with water, was treated with $10 \mu \mathrm{l} 10 \%$ DNFB in acetone and $100 \mu \mathrm{l}$ $4 \% \mathrm{NaOH}$. Fifteen minutes later, $100 \mu 110 \% \mathrm{H}_{3} \mathrm{PO}_{4}$ was added and the mixture was extracted with 500 $\mu \mathrm{CHCl}{ }_{3}$. The aqueous phase was extracted again with $50 \mu \mathrm{CHCl}_{3}$. It was found that DNP-taurine and DNP-cysteic acid were the only DNP derivatives in the reaction mixture after chloroform extraction. The pair of DNP derivatives was easily separated on a Silosorb $\mathrm{C}_{18}$ column with aqueous methanol containing $1 \%$ anhydrous acetic acid as mobile phase and detection at $350 \mathrm{~nm}$. The results obtained were compared with those obtained for the same samples using the amino acid analyser. The two techniques gave similar results.

\subsubsection{1-Dimethylaminonaphthalene-5-sulfonyl} chloride derivatization. Dansyl-Cl, as a well-known fluorogenic reagent for the determination of primary and secondary amines, was also reported in the HPLC determination of taurine in biological samples [6,56-58]. The strongly fluorescent dansyl-taurine derivative exhibits stability and chromatographic advantages compared with other chromophores. However, the derivatization reaction requires quenching, high temperatures and a long time. Amiss et al. [56] confirmed the suitability of dansylation for feline plasma and whole blood by thermospray mass spectrometry. Plasma or whole blood was diluted with a mixture of acetonitrile-methanol-triethylamine-water (25:22:3:50, v/v), filtered through a molecular mass 10000 exclusion filter, and then derivatized with dansyl-Cl. Excess dansyl-Cl favors the derivatization. The dansylation reaction, terminated by a $10-\mu l$ volume of a $3.5 \%(\mathrm{v} / \mathrm{v})$ aqueous solution of ethylamine, can be completed in $30 \mathrm{~min}$ at room temperature $\left(24^{\circ} \mathrm{C}\right)$. Increasing the reaction 
temperature did not improve the reaction efficiency and may increase its variability. Spiked plasma samples were reliably stable overnight $(\leq 18 \mathrm{~h})$ at room temperature, if protected from light. Dansyltaurine was separated from other compounds on a $\mathrm{C}_{18}$ column with a methanol-acetic acid-triethylamine (30:0.5:0.025) aqueous mobile phase. The effluent was monitored fluorimetrically at an excitation wavelength of $329 \mathrm{~nm}$ and an emission wavelength of $530 \mathrm{~nm}$. Although the method was very simple, both sample pretreatment and separation were time consuming (ca. 45 and $15 \mathrm{~min}$, respectively).

Based on the work of Amiss et al., Woollard and Indyk [6] developed a HPLC method for the evaluation of taurine in milk. The dansylation reaction, stopped by the addition of $100-\mu l$ methylamine hydrochloride solution, could be completed in $2 \mathrm{~h}$ in the dark at ambient temperature. Separation of the taurine derivatives was accomplished on a Waters $\mathrm{C}_{18}$ Resolve cartridge within 10 min without interference from other free amino acids using $0.1 \mathrm{~mol} / 1$ acetate buffer-acetonitrile-tetrahydrofuran (81:17:2, $\mathrm{v} / \mathrm{v}$ ) as mobile phase in a simple isocratic elution. The procedure was suitable for rapid data collection at sample concentrations above $1 \mathrm{mg}$ per $100 \mathrm{~g}$ and could be extended using fluorescence to assay products with lower taurine contents. UV detection was at $254 \mathrm{~nm}$ and fluorescence was monitored at $330 \mathrm{~nm}$ (excitation) and $530 \mathrm{~nm}$ (emission). The derivatized extract could be stored refrigerated at $4{ }^{\circ} \mathrm{C}$ in the dark, without degradation, for $48 \mathrm{~h}$ prior to analysis, which was a benefit for routine analysis.

To evaluate this isocratic HPLC separation of dansyl-derivative with UV and/or fluorescence detection, Woollard and Indyk [57] conducted a collaborative study. The statistical data confirmed the suitability of this method for the determination of taurine in both milk and infant formulae. They also found that fully protein-hydrolyzed formulae might occasionally be encountered, yielding chromatographic complications requiring gradient elution schemes for resolution of taurine.

McCarthy et al. [58] conducted another collaborative study to evaluate a method, based on a gradient HPLC separation of dansyl-derivative with fluorescence detection for the determination of total taurine in both wet and dry cat or dog food in a con- centration range of ca. $150-2400 \mathrm{mg} / \mathrm{kg}$. A $400-\mathrm{mg}$ amount of ground dry food or $800 \mathrm{mg}$ homogenized wet food was hydrolyzed to release bound taurine, and then derivatized with dansyl-Cl. The dansylation reaction was completed in $30 \mathrm{~min}$ at $65^{\circ} \mathrm{C}$. The method has been adopted Official First Action status by AOAC International.

\subsubsection{Fluorescamine derivatization. Fluores-} camine, first used for the derivatization of primary amines and amino acids in the early 1970s, has been described for the pre-column derivatization of taurine by HPLC with fluorescence [32] or UV absorbance detection [17]. Advantages of fluorescamine over all the above mentioned derivatizing reagents are that the reaction is instantaneous at ambient temperature in alkaline medium, the derivatives are stable in aqueous solution, whereas the unreacted reagent is rapidly hydrolysed to a product that does not interfere with the analysis. Using pre-column derivatization with fluorescamine and separation on a $\mathrm{Li}$ Chrospher 100 RP-8 column, Sakai and Nagasawa [32] first reported the plasma taurine levels of yellowtail fish $(125 \pm 54 \mu \mathrm{g} / \mathrm{ml})$, beef cattle $(5.6 \pm 1.4 \mu \mathrm{g} / \mathrm{ml})$, dairy cows $(2.2 \pm 0.7 \mu \mathrm{g} / \mathrm{ml})$ and chicken $(20.0 \pm 9.6 \mu \mathrm{g} / \mathrm{ml})$. The mobile phase consisted of $23 \%$ of acetonitrile in $15 \mathrm{mM}$ phosphate buffer ( $\mathrm{pH}$ 1.9). The derivatization reaction proceeded for $15 \mathrm{~min}$ at room temperature in the presence of $20 \mu \mathrm{l}$ of phosphate buffer $(200 \mu \mathrm{m}, \mathrm{pH}$ 7.8). Fluorescence was monitored at $400 \mathrm{~nm}$ for excitation wavelength and $480 \mathrm{~nm}$ for emission wavelength. The method required only $0.2 \mathrm{ml}$ of plasma and only $15 \mathrm{~min}$ of analysis time. However, there was discrepancy in the stability of taurinefluorescamine derivative.

McMahon et al. [17] found that the fluorescence intensity of taurine-fluorescamine derivative might decrease over a few hours whereas the absorbance remains unchanged for up to 1 week. Therefore, the derivative was by UV detected at $385 \mathrm{~nm}$ after separation on a Bondclone $\mathrm{C}_{18}$ column with tetrahydrofuran-acetonitrile-phosphate buffer $(15 \mathrm{mM}$, pH 3.5) $(4: 24: 72, \mathrm{v} / \mathrm{v})$ as mobile phase. The method was simple, efficient and rapid for the determination of taurine in platelet-rich plasma, with comparable sensitivity to fluorescence methods. 


\subsubsection{4-Dimethylaminoazobenzene-4'-sulfonyl} chloride derivatization. DABS-Cl has also been used for the pre-column derivatization of taurine for the high sensitivity, reliability and perfect reproducibility [2,35]. Stocchi et al. [2] presented a gradient HPLC method for the evaluation of taurine in human plasma samples with sensitivity at the picomol level. A $10-\mu 1$ volume of plasma sample, either extracted by $0.5 \mathrm{ml}$ of $5 \%$ perchloric acid or filtrated by CF 50 Amicon membrane, was derivatized with $40 \mu$ of DABS-Cl solution $(4 \mathrm{nmol} / \mu \mathrm{l}$ acetonitrile, freshly prepared) in the presence of $10 \mu \mathrm{l}$ of $0.1 \mathrm{~mol} / 1$ $\mathrm{NaHCO}_{3}, \mathrm{pH}$ 9. The reaction was performed at $70{ }^{\circ} \mathrm{C}$ for $10 \mathrm{~min}$. The derivatives were separated on a Supelcosil LC-18T column and then detected at 436 $\mathrm{nm}$. The analytical results showed that there was no difference between a perchloric acid extracted $(38 \pm 5$ $\mathrm{nmol} / \mathrm{l})$ and a filtrated plasma sample $(42 \pm 4 \mathrm{nmol} /$ 1). However, plasma filtration was a more rapid procedure, which also allowed the simultaneous evaluation of plasma acid-labile amino acids such as asparagines, glutamine and tryptophan. The recovery of taurine was higher than $96 \%$.

Hypotaurine-taurine status in animal body is important for the elucidation of the physiological roles of hypotaurine and taurine. Unfortunately, hypotaurine is unstable and is oxidized to taurine spontaneously or by ultraviolet irradiation. This instability of hypotaurine hindered the development of simple methods for the determination of hypotaurine. Two methods have been reported to successfully solve this problem $[23,35]$. One was by using DABS-Cl as the pre-column derivatizing reagent. Futani et al. [35] found that dabsylhypotaurine once formed was very stable, and it was not converted into dabsyltaurine on storage at room temperature for several months or on flash evaporation at $40{ }^{\circ} \mathrm{C}$. The oxidation of hypotaurine was minimized when dabsylation was performed at $40{ }^{\circ} \mathrm{C}$ in $65 \%$ acetone solution. The derivatives were separated on a TSKgel ODS-80Ts column at $16{ }^{\circ} \mathrm{C}$ and then detected at $430 \mathrm{~nm}$. The detection limit of both dabsylhypotaurine and dabsyltaurine was 4.0 pmol. Average excretions of taurine and hypotaurine in rat urine were 270.5 and $2.5 \mu \mathrm{mol} / \mathrm{kg}$ body mass per day, respectively. Another was by using 3,5-dinitrobenzoyl chloride as the derivatization reagent: 3,5 dinitrobenzoyl chloride is a useful reagent for the derivatization of alcohols and amines, yielding strongly UV-absorbing products. Masuoka et al. [23] treated aqueous sample solution containing taurine and hypotaurine with freshly powdered 3,5-dinitrobenzoyl chloride in the presence of triethylamine. The N-3,5-dinitrobenzoyl derivatives were stable enough for analysis. About $5 \%$ of the hypotaurine derivative was oxidized by oxygen in the air to give $\mathrm{N}$-(3,5-dinitrobenzoyl)taurine when the derivatized sample was left at room temperature for 2 days. The derivatives were separated on a TSKgel ODS-80Ts column with a mobile phase of $16 \%$ acetonitrile in $100 \mathrm{~m} M$ ammonium acetate buffer (pH 3.7) and then detected at $254 \mathrm{~nm}$. The detection limits for both compounds were $0.5 \mu \mathrm{mol}$. Other amino acids and ammonium could be simultaneously analyzed by the present method.

\subsubsection{Ion chromatography}

Ion chromatography (IC) was a powerful technique for the analysis of inorganic cations and anions in aqueous systems. However, with the development of IC technique went on, some IC methods progressed to organic ion problem solving. The main advantage of IC methods over HPLC methods for the analysis of taurine is that it need no pre- or postcolumn derivatization steps, which is beneficial for routine analysis. Usually, weakly ionized short chain organic acids are separated by ion-exclusion chromatography with suppressed conductivity detection. Since the dissociation constant $\mathrm{p} K_{\mathrm{a}}$ (4.96) of taurine is too large, it cannot be detected sensitively with conductivity detection. It cannot be detected with UV detection due to the lack of an ultraviolet chromophore. Zhu and co-workers [59,60] showed that taurine, leucine and phenylalanine could be separated on an IonPac AG4A-SC4 column with $0.75 \mathrm{~m} M \mathrm{KCl}-\mathrm{NaOH}$ of $\mathrm{pH} 11.5$ as mobile phase, followed by indirect suppressed conductivity detection. There was no interference from sulfate, chloride and phosphate, etc. The detection limits for taurine, leucine and phenylalanine were $0.53,0.38$ and 8.3 $\mathrm{mg} / \mathrm{l}$, respectively. The sensitivity of suppressed conductivity detection of taurine is not high compared with HPLC methods. No real sample analysis was performed.

$\mathrm{Qu}$ et al. [8] first reported an IC method with electrochemical integrated pulsed amperometry de- 
tection to determine the taurine levels in medicine, nutrient capsule and human urine. They selected CarboPac PA1 column as the separation column, which has both ion-exchange functional group and significant hydrophobic character. It is the combination of anion-exchange and hydrophobic interaction that gives the incredible power for retaining the carbohydrate compounds, which have very high $\mathrm{p} K_{\mathrm{a}}$ values and are poorly retained on other anion-exchange columns. Taurine was thus well separated from 10 kinds of common amino acids using 160 $\mathrm{m} M \mathrm{NaOH}$ as the eluent. The chromatogram of taurine in human urine is shown in Fig. 3. The detection limit of taurine is $0.034 \mu \mathrm{g} / \mathrm{ml}$, which is comparable with that of HPLC methods.

\subsubsection{Thin-layer chromatography (TLC)}

In general, TLC is simple and does not require special equipment. However, It was rarely applied to the analysis of taurine in biological samples due to its low sensitivity and reproducibility compared with HPLC. Until now, two papers dealing with the analysis of taurine in "energy drinks" have been reported [61,62].

\subsubsection{Gas chromatography (GC)}

There are also a limited number of publications involving the use of GC for the analysis of taurine.

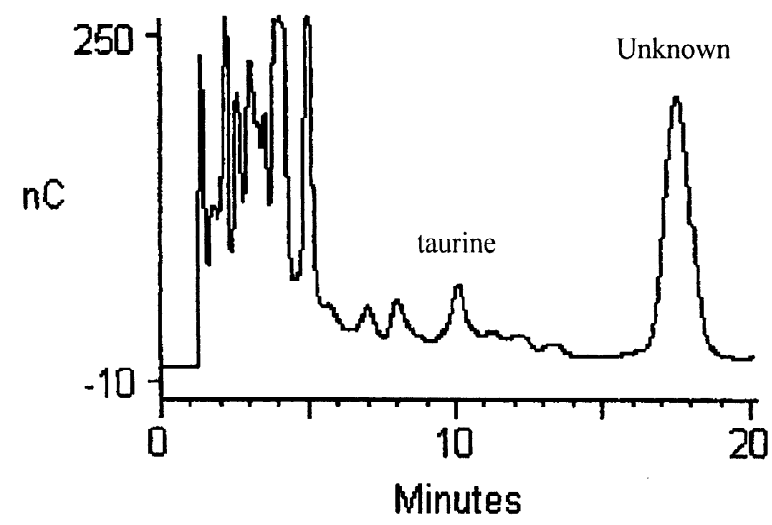

Fig. 3. Ion chromatogram of urine sample. Column: CarboPac PA1 separation column $(250 \times 4 \mathrm{~mm}$ I.D. $)$ and PA guard column ( $25 \times 4 \mathrm{~mm}$ I.D.); eluent: $160 \mathrm{mM} \mathrm{NaOH}$; flow-rate: $1 \mathrm{ml} / \mathrm{min}$; detection: Electrochemical in the electrochemical integrated amperometry detection mode. (Taken from Ref. [8] with permission).
The main difficulty of taurine analysis using GC is the formation of a volatile derivative of the sulfonic function. Stämpfli et al. [63] used the same derivative- $N$-pentafluorobenzoyl di- $n$-butyl amide as Kataoka et al. [64] described, and slightly modified the derivative procedure to complete the measurement of ${ }^{15} \mathrm{~N}$ taurine in cat urine by gas chromatography-electron impact mass spectrometry (GC-EIMS). The result showed that $85 \%$ of cat urinary taurine was in the free form. Marks et al. [16] confirmed the validity of the GC-EI-MS technique and extended the use of this technique to human plasma. The plasma taurine concentration was found to be $56 \pm 14 \mu \mathrm{mol} / \mathrm{l}$, which was agreed well with available literature values determined by the commonly used high-performance liquid chromatographic method.

\subsection{Electro-migration methods}

Capillary electrophoresis (CE) is the mainly used electro-migration (electrophoresis) method for taurine analysis. It is an analytical counterpart to HPLC and offers a number of advantages over HPLC: very little organic solvent in the running buffer, short run time for the separation, high separation efficiencies, $10^{6}$ plates $/ \mathrm{m}$, etc. However, $\mathrm{CE}$ has not been widely applied to the analysis of taurine in biological samples due to the complexity of biological samples. At present, seven researches $[10,18,22,65-67]$ dealing with the analysis or identification [68] of taurine in biological samples have been reported.

Among the above mentioned pre-column derivatization reagents, only fluorescamine is a suitable derivatization regent for $\mathrm{CE}$ analysis. Kelly et al. [18] developed a rapid and highly selective capillary zone electrophoresis (CZE) method for the determination of taurine in plasma. The separation is based on the difference in ionization of taurine from that of other amino acids due to the fact that taurine is a sulfonic rather than a carboxylic amino acid. Homotaurine was selected as internal standard because it had the better handling properties than aminomethane sulfonic acid. Following addition of homotaurine, plasma proteins were precipitated with acetonitrile and the supernatant was derivatized with fluorescamine in the presence of a borate buffer. CE 
separations were carried out in the reverse polarity mode at $27.5 \mathrm{kV}$ with a diode array detector set at $266 \mathrm{~nm}$ (Fig. 4). A capillary conditioning solution was applied daily in order to suppress the residual electroosmotic flow. The relative standard deviations (RSDs) of replicate analysis were less than $4.5 \%$ at 1 $\mu \mathrm{g} / \mathrm{ml}$ taurine in feline plasma and less than $3 \%$ for $2.5 \mu \mathrm{g} / \mathrm{ml}$ in human plasma. Recovery was estimated at $99.2 \%$ with an RSD of $4.85 \%$.

Jellum et al. [10] measured taurine levels at submilligram samples of biopsy of human heart muscle to diagnose and study human disease, particularly metabolic disorders. The samples were homogenized in phosphate buffer of $\mathrm{pH} 7.5$ and then deproteinized by perchloric acid. The supernatant was neutralized with trisodium phosphate solution and then derivatized with 9-fluorenylmethyl chloroformate (FMOC) for $20 \mathrm{~min}$ at room temperature. The derivatized taurine was well separated from 3-amino-1-propane-

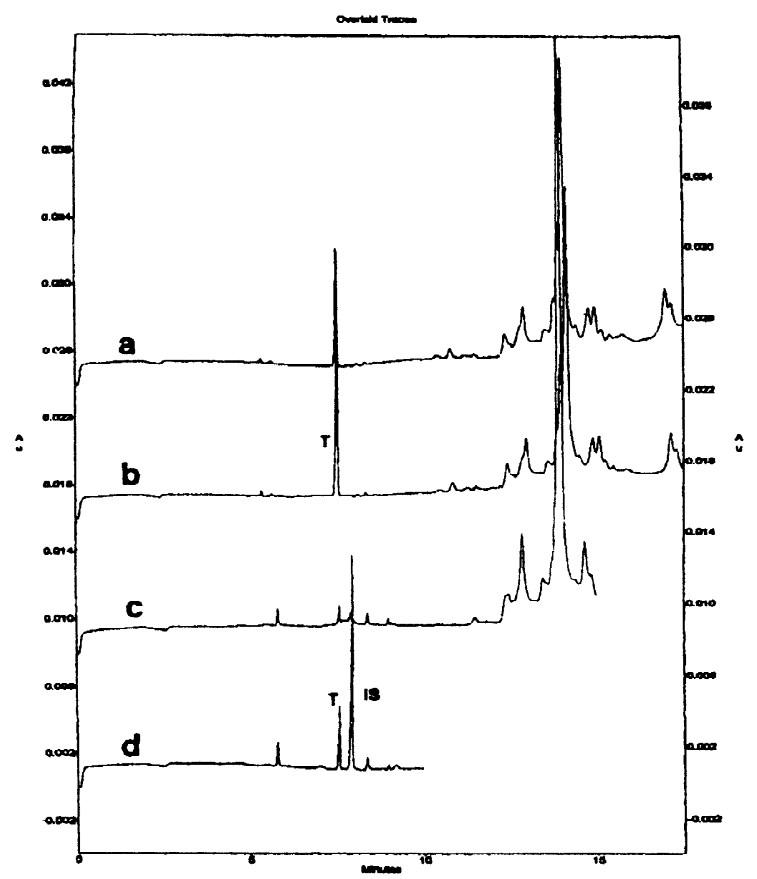

Fig. 4. Electropherograms of (a) blank human plasma; (b) human plasma spiked with $5 \mu \mathrm{g} / \mathrm{ml}$ taurine (T); (c) blank feline plasma and (d) feline plasma spiked with $5 \mu \mathrm{g} / \mathrm{ml} \mathrm{T}$ and $20 \mu \mathrm{g} / \mathrm{ml}$ homotaurine (I.S.). Fused-silica capillary: $52.0 \mathrm{~cm} \times 75 \mu \mathrm{m}$ I.D.; temperature: $20{ }^{\circ} \mathrm{C}$; sample tray temperature: $5^{\circ} \mathrm{C}$; detection: diode array at $266 \mathrm{~nm}$. (Taken from Ref. [18] with permission). sulfonic acid (internal standard) and glutathione by using $0.05 \mathrm{~mol} / 1$ sodium phosphate $(\mathrm{pH} 2.5)$ as running buffer, and then sensitively detected by fluorescence spectroscopy.

Another procedure has appeared for the simultaneous determination of six amino acid neurotransmitters including taurine in the dorsal root ganglion of rat by $\mathrm{CE}$ with a laser-induced fluorescencecharge coupled device (LIF-CCD) [22]. The homogenated samples were acidified with concentrated perchloric acid and then centrifuged. The supernatants were derivatized with fluorescein isothiocyanate (FITC) at $40^{\circ} \mathrm{C}$ for $6 \mathrm{~h}$. The derivatives were then separated on a fused-silica capillary at $12.5 \mathrm{kV}$ with $15 \mathrm{~m} M$ borate (pH 9.2) as the running buffer. A unique feature of the method was that CCD was an image detector with a matrix array of photoelements, the CCD readouts from single photoelements could be summed up by on-chip analog summation called charge binning, or by digital summation in the computer. Hence, the detection sensitivity could be improved dramatically. The detection limits ranging from 1.2 to $17.2 \mathrm{n} M$ was obtained for the six neurotransmitters.

The fluorogenic reagent 5-furoylquinoline-3-carboxaldehyde (FQ) has proven valuable in the analysis of proteins and small neurotransmitters. Wu et al. [65] studied the reaction rate, activation energy, and detection limit for the reaction of FQ with five neurotransmitters including taurine in artificial cerebrospinal fluid. A reaction time of nearly $1 \mathrm{~h}$ was required to quantitatively convert these neurotransmitters to their fluorescent products at $65{ }^{\circ} \mathrm{C}$. Detection limits for the labeled neurotransmitters were $10^{-9}-10^{-8} M$, which corresponded to 0.3-7 amol injected onto the capillary. No real samples were analyzed.

Lillard et al. [68] characterized and identified amino-containing components including taurine that had not been determined previously in atrial gland vesicles by CE-LIF. Amino-containing components were on-line derivatized with naphthalene-2,3-dicarboxaldehyde (NDA), and then separated on a bare fused-silica capillary. Although the NDA derivatives are stable, but the reaction requires a cyanide salt which is toxic to the environment. The presence of taurine was confirmed unambiguously in lysed vesicles using HPLC, nuclear magnetic resonance 
(NMR), CE (with LIF and absorbance), and electrospray ionization mass spectrometry (ESI-MS).

Osaka et al. [66] presented a CZE method for analyzing nine major components (pyridoxine hydrochloride, neostigmine methylsulfate, chlorpheniramine maleate, allantoin, naphazoline hydrochloride, glycyrrhizic acid, chondroitin sulfate, taurine and cyanocobalamin) in eye lotion. Ferulic acid was used as internal standard. The samples were separated on a fused-silica capillary operated at $25 \mathrm{kV}$ with 20 $\mathrm{m} M$ borate buffer of $\mathrm{pH} 10$ as run buffer and detection at $200 \mathrm{~nm}$. It was found that cyanocobalamin could not be determined because its migration time was the same as electroosmotic flow; chondroitin sulfate and aspartic acid were resolved at $\mathrm{pH}$ 9. Although cyanocobalamin could be separated from other components by micellar electrokinetic chromatography (MEKC) using $20 \mathrm{~m} M$ borate of $\mathrm{pH}$ 10 containing $40 \mathrm{~m} M$ sodium dodecyl sulfate (SDS), it was difficult to separate several ingredients in eye lotion simultaneously by a single MEKC method.

MEKC can separate not only ionic but also electrically neutral compounds. The simultaneous determination of ingredients in ophthalmic solutions is particularly required to keep the quality of the preparations high. Therefore, Okamoto et al. [67] described the separation of 11 ingredients used in ophthalmic solutions by a mixed carrier system containing SDS with dimethyl- $\beta$-cyclodextrin (CD) and tetraalkylammonium phosphate (TAA) in CDMEKC. They investigated the effects of five different $C D$ types in the system on the separation and discussed the possible separation mechanism. Quantitation limits ranged from 1 to $80 \mu \mathrm{g} / \mathrm{ml}$ and the RSD values ranged from 4.1 to $6.0 \%$. The method is suitable for the simultaneous quantitative analysis as well as quantitative analysis in pharmaceutical quality control.

The derivatization, separation and determination methods for typical biological samples are summarized in Table 1. The actual concentrations of taurine in body fluids of some healthy and unhealthy persons are listed in Table 3.

\subsection{Hyphenation procedures}

Yoshizawa et al. [69] presented a hyphenation method for the determination of taurine levels in cosmetic and pharmaceutical preparations by highperformance liquid chromatography with inductively-coupled plasma atomic emission spectrophotometry (ICP-AES). Samples $(10 \mu \mathrm{l})$ were separated on a Capcell Pak $\mathrm{NH}_{2}$ column at $40{ }^{\circ} \mathrm{C}$ with $2 \mathrm{mM}$ $\mathrm{NH}_{4} \mathrm{H}_{2} \mathrm{PO}_{4}-\mathrm{NH}_{3}$ buffer (pH 8)-methanol (4:1) as

Table 3

The concentrations of taurine in body fluids of healthy and unhealthy persons

\begin{tabular}{lll}
\hline & Concentration $(\mathrm{nmol} / \mathrm{ml})$ & Ref. \\
\hline Healthy sample & & {$[2]$} \\
Plasma & $38 \pm 5 / 42 \pm 4$ & {$[15]$} \\
Plasma & $38-197$ & {$[16]$} \\
Plasma & $46 \pm 11-72 \pm 9$ & {$[19]$} \\
Plasma & $63 \pm 6$ & {$[17]$} \\
Platelet-rich plasma & $127.9-151.8$ & {$[17]$} \\
Platelet-poor plasma & $44.7 \pm 4$ & {$[34]$} \\
Urine & $778.9 \pm 71$ & {$[8]$} \\
Urine & $75.1-998.9$ & {$[23]$} \\
Blood & $159 \pm 19$ & {$[30]$} \\
Serum & $102.5 \pm 17.3$ & {$[34]$} \\
Human milk & $332.4 \pm 136.9$ & {$[14]$} \\
CSF & $6.54 \pm 1.32$ & {$[13]$} \\
CSF & $6.5 \pm 1.78$ & {$[23]$} \\
Blood & $159 \pm 19$ \\
Unhealthy sample & \\
Plasma (diabetic patients with poor metabolic control) & \\
Serum (patients with progressing ischemic stroke) & $60 \pm 5$ \\
\hline
\end{tabular}


mobile phase and cross-flow-nebulization ICP-AES detection at the $\mathrm{S} 180.73 \mathrm{~nm}$ emission line. The detection limit was $43 \mathrm{ng}$ and the RSDs were $\leq 2.2 \%$. Vonach et al. [70] coupled HPLC with Fourier transform infrared spectroscopy (FT-IR) to determine sugars in nonalcoholic beverage. The advantage of the flow-cell-based HPLC-FT-IR was that the combination of retention time and spectrum allowed an easy and unequivocal identification. However, both absorption in the fingerprint region and an analyte concentration in the milligrams-permilliliter range were required. The method was also suitable to identify and quantify minor components in beverages, such as taurine and ethanol.

\section{Conclusion}

(1) The delay between the sampling and preparation of a biological sample should be as short as possible to prevent variations in concentration of taurine due to metabolism.

(2) It is strongly recommended that the biological samples be deproteinized immediately after collection. If this is impossible, the samples should be stored at $-80{ }^{\circ} \mathrm{C}$ to prevent further hydrolysis of proteins.

(3) Acetonitrile, TCA and SSA have been successfully used as protein remover for various biological samples.

(4) The major chromatographic method for taurine analysis in biological samples, which has been frequently used during the last twelve years, is HPLC due to its high sensitivity, reliability and the relatively short time. However, most of them require complicated derivatization procedures due to the fact that taurine lacks an ultraviolet chromophore.

(5) Ion chromatographic method with electrochemical integrated amperometry detection makes pre- or post-column derivatization unnecessary and shows good sensitivity and selectivity, which has a broad future in routine analysis of taurine in biological samples.

(6) Derivatizing reagents used for HPLC methods are different from those used for CE methods with exceptions of fluorescamine. CE method with precapillary derivatization is a good alternative to the HPLC method for the taurine analysis of plasma.
However, there is still some room for improvement from the viewpoint of reproducibility.

(7) Hyphenation techniques, such as HPLC-FTIR and HPLC-ICP-AES have been proved to be effective for the analysis of taurine in biological samples. However, they have not been widely used due to the complex apparatus.

\section{References}

[1] D.L. Hardy, J. Norwood, J. Magn. Reson. 133 (1998) 70.

[2] V. Stocchi, F. Palma, G. Piccoli, B. Biagiarelli, L. Cucchiarini, M. Magnani, J. Liq. Chromatogr. 17 (2) (1994) 347.

[3] I.M.P.L.V.O. Ferreira, M.V. Nunes, E. Mendes, F. Remião, M.A. Ferreira, J. Liq. Chromatogr. Rel. Technol. 20 (8) (1997) 1269.

[4] R.C. Chen, Nutrition and Health Food, China Light Industry Press, 2001, p. 161.

[5] C.J. Waterfield, J. Chromatogr. B 657 (1994) 37.

[6] D.C. Woollard, H.E. Indyk, Food Chem. 46 (1993) 429.

[7] E.C. Nicolas, K.A. Pfender, M.A. Aoun, J.E. Hemmer, J. Assoc. Off. Anal. Chem. 73 (1990) 627.

[8] F. Qu, Z.H. Qi, K.N. Liu, S.F. Mou, J. Chromatogr. B 730 (1999) 161.

[9] D. Fekkes, A.V. Kooyman, R. Jankie, J. Huijmans, J. Chromatogr. B 744 (2000) 183.

[10] E. Jellum, A.K. Jhorsrud, E. Time, J. Chromatogr. 559 (1991) 455.

[11] A.A. Stämpfli, O. Ballèvre, L.B. Fay, Rapid Commun. Mass Spectrom. 6 (1992) 547.

[12] F. Couderc, E. Caussé, N. Siméon, C. Bayle, M. Nertz, B. Feurer, Am. Lab. February (2001) 46.

[13] V. Rizzo, A. Anesi, L. Montalbetti, G. Bellantoni, R. Trotti, G.V. Melzi d'Eril, J. Chromatogr. A 729 (1996) 181.

[14] A. Anesi, M. Rondanelkli, G. Melzi d'Eril, Clin. Chem. 44 (1998) 2359

[15] T. Teerlink, P.A.M. van Leeuwen, A. Houdijk, Clin. Chem. 40 (2) (1994) 245.

[16] L. Marks, F. Iglicki, B. Rakotoambinina, F. Thuillier, B. Messing, J. Mass Spectrom. 30 (1995) 1687.

[17] G.P. McMahon, R. O'Kennedy, M.T. Kelly, J. Pharm. Biomed. Anal. 14 (8-10) (1996) 1287.

[18] M.T. Kelly, H. Fabre, D. Perrett, Electrophoresis 21 (2000) 699.

[19] V. Fierabracci, P. Masiello, M. Novelli, E. Bergamini, J. Chromatogr. 570 (1991) 285.

[20] S. Murai, H. Saito, Y. Masuda, T. Itoh, J. Pharmacol. Methods 23 (1990) 195.

[21] T. Ide, J. Chromatogr. B 694 (1997) 325.

[22] L. Zhang, H. Chen, S. Hu, J. Cheng, Z.W. Li, M. Shao, J. Chromatogr. B 707 (1998) 59.

[23] N. Masuoka, K. Yao, M. Kinuta, J. Oha, M. Wakimoto, T. Ubuka, J. Chromatogr. B 660 (1994) 31. 
[24] Y. Qu, L. Arckens, E. Vandenbussche, S. Geeraerts, F. Vandesande, J. Chromatogr. A 798 (1998) 19.

[25] M. Gupta, M.K.P. Amma, J. Liq. Chromatogr. 15 (12) (1992) 2153

[26] P.L. Weber, T.J. O'Shea, S.M. Lunte, J. Pharm. Biomed. Anal. 12 (1994) 319.

[27] M.C. Aristoy, F. Toldrá, J. Agric. Food Chem. 39 (1991) 1792.

[28] B. Polanuer, S. Ivanov, A. Sholin, J. Chromatogr. B 656 (1994) 81.

[29] T. Yokoyama, T. Kinoshita, J. Chromatogr. 568 (1991) 212.

[30] Y.V. Tcherkas, L.A. Kartsova, I.N. Krasnova, J. Chromatogr. A 913 (2001) 303.

[31] A.M. Uhe, G.R. Collier, E.A. Mclennan, D.J. Tucker, K. Q'Dea, J. Chromatogr. 564 (1991) 81.

[32] T. Sakai, T. Nagasawa, J. Chromatogr. 576 (1992) 155.

[33] A. Schmid, G. Sperk, H. Reither, Comp. Biochem. Physiol. 102C (1992) 447.

[34] Z.L. Chen, G. Xu, K. Specht, R.J. Yang, S.W. She, Anal. Chim. Acta 296 (1994) 249.

[35] S. Futani, T. Ubuka, T. Abe, J. Chromatogr. B 660 (1994) 164.

[36] S. Yuasa, R. Akagi, T. Ubuka, Acta Med. Okayama 44 (1990) 47.

[37] C.G. Zhou, Chin. J. Chromatogr. 9 (1991) 274.

[38] C.G. Zhou, W. Sun, Chin. J. Chromatogr. 11 (1993) 187.

[39] J. Le-Boucher, C. Charret, C. Coudray-Lucas, J. Giboudeau, L. Cynober, Clin. Chem. 43 (1997) 1421.

[40] T. Yokoyama, T. Kinoshita, Bunseki Kagaku 40 (1991) 349.

[41] K. Saito, M. Horie, Y. Tokumaru, H. Nakazawa, Shokuhin Eiseigaku Zasshi 38 (6) (1997) 400.

[42] P. Furst, L. Pollack, T.A. Graser, H. Godel, P. Stehle, J. Chromatogr. 499 (1990) 557.

[43] V.R. Roettger, M.D. Goldfinger, J. Neurosci. Methods 39 (1991) 263

[44] I.N. Krasnova, L.A. Kartsova, Y.V. Cherkas, Zh. Anal. Khim. 55 (2000) 58.

[45] R.S. Ersser, J.F. Davey, Med. Lab. Sci. 48 (1991) 59.

[46] Z. Yang, P. Zhang, Chin. J. Health Res. 27 (3) (1998) 192.

[47] G. Zheng, S.B. Lin, Chin. J. Health Res. 27 (3) (1998) 266.

[48] L. Bianchi, L. Della Corte, K.F. Tipton, J. Chromatogr. B 723 (1999) 47.
[49] C.S. Yang, P.J. Tsai, W.Y. Chen, W.J. Tsai, J.S. Kuo, J. Chromatogr. B 734 (1999) 1.

[50] T.P. Piepponen, A. Skujins, J. Chromatogr. B 757 (2001) 277.

[51] H. Xie, S. Zhang, Chin. J. Chromatogr. 15 (1997) 54.

[52] H.L. Rowley, K.F. Martin, C.A. Marsden, J. Neurosci. Methods 57 (1995) 93.

[53] J.H. Xie, Y.R. Chen, S.Z. Tang, Chin. J. Chromatogr. 12 (4) (1994) 301.

[54] Z.G. Tang, R. Q Zhou, Z.T. Duan, Chin. J. Anal. Chem. 27 (1999) 1084.

[55] M. Polanuer, S.V. Ivanov, A.F. Sholin, Khim. Farm. Zh. 29 (1995) 52.

[56] T.J. Amiss, K. Tyczkowska, D.P. Aucoin, J. Chromatogr. 526 (1990) 375.

[57] D.C. Woollard, H.E. Indyk, J. AOAC Int. 80 (1997) 860.

[58] K. McCarthy, C. Hischenhuber, N. Joyce, J. AOAC Int. 83 (2000) 784.

[59] Y. Zhu, S.F. Wang, W.P. Liu, LC·GC North America 18 (2000) 200.

[60] Y. Zhu, S.F. Wang, Chin. J. Anal. Sci. 16 (2000) 176.

[61] A. Kotzbauer, P. Pfeifer, T. Roedor, GIT-Fachz-Lab 40 (1996) 619.

[62] G. Indrayanto, T.K. Sia, Y.I. Wibowo, J. Planar Chromatogr. Mod. TLC 14 (2001) 24.

[63] A.A. Stämpfli, O. Ballevre, L.B. Fay, J. Chromatogr. 617 (1993) 197.

[64] H. Kataoka, N. Ohnishi, M. Makita, J. Chromatogr. 339 (1985) 370.

[65] J. Wu, Z. Chen, N.J. Dovichi, J. Chromatogr. B 741 (2000) 85.

[66] S. Osaka, Y. Sawabe, T. Nakagawa, Jpn. J. Toxicol. Environ. Health 41 (1995) 85.

[67] H. Okamoto, A. Uetake, R. Tamaya, T. Nakajima, K. Sagara, Y. Ito, J. Chromatogr. A 888 (2000) 299.

[68] S.J. Lillard, D.T. Chiu, R.H. Scheller, R.N. Zare, Anal. Chem. 70 (1998) 3517.

[69] D. Yoshizawa, S. Yamamoto, K. Ishiwata, T. Takamatsu, M. Matsuoka, Bunseki Kagaku 44 (1995) 603.

[70] R. Vonach, B. Lendl, R. Kellner, Anal. Chem. 69 (1997) 4286. 